\title{
Experiencia en niños de la gastrostomía laparoscópica por miniestoma
}

\author{
Édgar Salamanca ${ }^{1}$, Juan Enrique Sebán ${ }^{1}$, Andrea Suárez ${ }^{2}$
}

Palabras clave: gastrostomía; laparoscopía; pediatría; nutrición entérica; complicaciones posoperatorias.

\begin{abstract}
Resumen
Introducción. El presente artículo tiene como fin describir la experiencia de la gastrostomía por laparoscopia por medio de la técnica de miniestoma, con algunas modificaciones, en dos hospitales de cuarto nivel de Bogotá.

Materiales y métodos. Se llevó a cabo un estudio de casos en el grupo de pacientes pediátricos (0 a 13 años), que fueron sometidos a gastrostomía por laparoscopia con la técnica de miniestoma, del $1^{\circ}$ de agosto de 2013 al 31 de enero de 2015. Se describen los factores que podrían influir en la seguridad del paciente antes, durante o después de la cirugía.
\end{abstract}

Resultados. Se practicaron 47 gastrostomías en pacientes entre 1 mes y 13 años de edad. La indicación principal para la gastrostomía fue el trastorno de succión-deglución (44/47; $93 \%$ ). Las enfermedades que presentaban los pacientes fueron $85 \%$ de origen neurológico y $57 \%$ de

1 Cirujano pediatra, Departamento de Cirugía Pediátrica, Fundación Cardioinfantil, Hospital Universitario San Ignacio, Bogotá, D.C., Colombia

2 Médica general, investigadora, Departamento de Cirugía Pediátrica, Fundación Cardioinfantil, Bogotá, D.C., Colombia

Fecha de recibido: 10 de enero de 2017

Fecha de aprobación: 7 de julio de 2017

Citar como: Salamanca E, Sebá JE, Suárez A . Experiencia en niños de la gastrostomía laparoscópica por miniestoma. Rev Colomb Cir. 2017;32:176-81. origen cardiaco. El 89,36\% de los dispositivos utilizados fueron retenedores inflables. La nutrición entérica se inició en las primeras 24 horas en la mayoría de los pacientes, sin que se documentaran complicaciones relacionadas. La mediana del tiempo de duración del procedimiento fue de 57 minutos. Solo se documentaron complicaciones posoperatorias menores.

Discusión. La gastrostomía por laparoscopia tiene múltiples ventajas: es un procedimiento seguro, reproducible y solo requiere instrumental básico de laparoscopia por lo que es recomendable en la población pediátrica.

\section{Introducción}

Los pacientes pediátricos con trastornos neurológicos, enfermedades de las vías digestivas y cardiopatías congénitas que antes fallecían tempranamente, hoy sobreviven gracias a los avances tecnológicos y científicos en el cuidado neonatal, las unidades de cuidados intensivos y los métodos de soporte nutricional. Algunos de estos niños requieren una gastrostomía para su alimentación en las etapas tempranas de la vida.

Inicialmente, el abordaje de estos pacientes se hacía con técnicas abiertas, como la gastrostomía de Stamm ${ }^{1}$. En 1980 aparecieron otras opciones, cuando Gauderer y Ponsky ${ }^{2}$ describieron la gastrostomía endoscópica percutánea, la cual tuvo mucha aceptación por ser mínimamente invasiva, sin tener un costo mayor ${ }^{3}$. En 1991, Edelman ${ }^{4}$ reportó por primera vez la experiencia con el abordaje de la gastrostomía por laparoscopia, en la cual se inserta el tubo de gastrostomía, bajo visión directa y de una forma segura ${ }^{5}$. 
Para practicar la gastrostomía laparoscópica, se ha descrito la técnica Seldinger y la técnica de miniestoma (mini-open technique), cuya principal diferencia es la forma de realizar el estoma. En la técnica de Seldinger, el estómago se fija a la pared abdominal con puntos en $\mathrm{U} y$, mediante un dispositivo especial, se abre el orificio de la gastrostomía por vía percutánea. En la técnica de miniestoma, el estómago se exterioriza, se abre bajo visión directa y se ajusta por medio de una jareta ${ }^{6}$.

Se describe la experiencia con la gastrostomía por laparoscopia con la técnica de miniestoma, sus resultados y las complicaciones más frecuentes, en niños de dos hospitales de cuarto nivel de Bogotá. Además, se describe la técnica quirúrgica utilizada y se aportan algunos detalles que facilitan la cirugía.

\section{Materiales y métodos}

Se trata de una serie de casos de un grupo de pacientes pediátricos de 0 a 13 años de edad, a quienes se les practicó gastrostomía laparoscópica en el periodo del $1^{\circ}$ de agosto de 2013 al 31 de enero de 2015 en la Fundación Cardioinfantil y el Hospital Universitario San Ignacio de Bogotá.

Se revisaron las historias clínicas de los pacientes de interés y se caracterizó la población en términos de sexo, edad, enfermedad, indicación de la gastrostomía, tipo de dispositivo utilizado, tiempo quirúrgico, necesidad de cuidados intensivos, número de días en iniciar la nutrición entérica, complicaciones, razón y tiempo del primer recambio de dispositivo, y resultados como muerte y necesidad de cierre de la gastrostomía.

Las complicaciones se agruparon en transoperatorias, si se produjeron durante la cirugía; posoperatorias, si se identificaron después del acto quirúrgico, y asociadas o no al cuidado de la gastrostomía.

La técnica quirúrgica utilizada fue la de miniestoma -con pequeñas variaciones-, la cual se describe a continuación.

Se hace una incisión transumbilical y se coloca un puerto de 3 o $5 \mathrm{~mm}$ con técnica abierta para el paso del lente. Se produce el neumoperitoneo mediante insuflación de $\mathrm{CO}_{2}$ con presiones de 6 a $10 \mathrm{mmHg}$ y flujo de 5 litros por minuto. Se identifica el estómago bajo visión laparoscópica insuflando aire a través de la sonda nasogástrica.

Se hace una incisión en el cuadrante superior izquierdo para pasar el puerto de 3 a $5 \mathrm{~mm}$, se toma con una pinza el sitio de la pared gástrica determinado para colocar la gastrostomía y se exterioriza, retirando el puerto. Se fija haciendo una jareta con sutura PDS $4-0^{\circledR}$ o Vicryl 4- $0^{\circledR}$; se hace la incisión gástrica bajo visión directa y se fija la pared gástrica a la pared abdominal mediante sutura de puntos separados. Se coloca la sonda de gastrostomía y se cierra la jareta.

Bajo visión laparoscópica, se verifica la posición intragástrica del dispositivo, insuflando aire a través de la sonda nasogástrica y extrayéndolo por el dispositivo de gastrostomía. En el período posoperatorio inmediato, se dejó libre el drenaje por el dispositivo de la gastrostomía, y la alimentación entérica se inició en el primer día.

\section{Resultados}

Se practicaron 47 gastrostomías en 31 niños y 16 niñas, con una mediana de edad de seis meses, y un rango entre un mes y 13 años.

La indicación principal para la gastrostomía fue el trastorno de succión-deglución en 44 de los 47 niños (93\%); en los tres restantes, las indicaciones fueron estenosis esofágica secundaria a ingestión de una pila de botón, desnutrición asociada a enfermedad cardiaca y mucositis secundaria a quimioterapia.

De los 47 pacientes, 40 (85\%) presentaban compromiso neurológico y 27 (57\%), una o más enfermedades cardíacas (tabla 1).

Los dispositivos de gastrostomía colocados fueron 42 botones de retenedor inflable, tres sondas de retenedor inflable y dos botones de retenedor elástico.

A $10(21 \%)$ de los pacientes se les practicó una intervención adicional a la gastrostomía durante el mismo acto quirúrgico: herniorrafia inguinal en dos, herniorrafia umbilical en dos, traqueostomía en dos, herniorrafia diafragmática en uno, nasofibrobroncoscopia en uno, liberación de adherencias en uno y dilatación anal en uno. 
TABLA 1.

Enfermedades cardiacas de los pacientes a quienes se les practicó gastrostomía

\begin{tabular}{ll}
\hline Enfermedad cardiaca más relevante & n \\
\hline Comunicación interventricular & 8 \\
Comunicación interauricular & 3 \\
Canal aurículo-ventricular & 2 \\
Coartación aórtica & 2 \\
Conducto arterial persistente & 2 \\
Síndrome de corazón izquierdo hipoplásico & 2 \\
Tetralogía de Fallot & 2 \\
Tronco arterial común & 2 \\
Atresia tricuspídea & 1 \\
Cardiomiopatía hipertrófica & 1 \\
Drenaje venoso anómalo & 1 \\
Transposición de grandes vasos & 1 \\
\hline
\end{tabular}

La mediana del tiempo quirúrgico fue de 57 minutos (desviación estándar, $\mathrm{DE}=22,03$ ), en los 37 pacientes a quienes solo se les practicó gastrostomía por laparoscopia.

De los 47 pacientes sometidos a gastrostomía, 19 (40\%) estuvieron en hospitalización general, de los cuales dos debieron ser trasladados a la unidad de cuidados intensivos después del procedimiento quirúrgico, uno de ellos porque se le practicó simultáneamente una corrección de hernia diafragmática, y el otro, por su enfermedad cardiaca de base y las intervenciones quirúrgicas cardiovasculares previas. Los $28(60 \%)$ restantes ya se encontraban en la unidad de cuidados intensivos y regresaron allí después de la gastrostomía.

Después de la gastrostomía, la mediana del tiempo en que se inició la vía entérica fue de un día $(\mathrm{DE}=0,83)$.

No se documentaron complicaciones durante la cirugía ni después de ella. Ninguno de los pacientes requirió conversión a cirugía abierta. En 19 (40 \%) pacientes se presentaron 27 complicaciones menores relacionadas con el cuidado de la gastrostomía (tabla 2). Se presentaron tres casos de infección, dos de estos en los primeros 30 días posoperatorios (días 10 y 16), los cuales se resolvieron con tratamiento antibiótico.

Durante el seguimiento, se presentaron ocho muertes, ninguna de ellas relacionada directamente con la
TABLA 2.

Complicaciones menores en niños a quienes se les practicó gastrostomía

\begin{tabular}{ll}
\hline Complicación & n \\
\hline Fuga & 9 \\
Eritema perigastrostomía & 6 \\
Granuloma perigastrostomía & 3 \\
Infección & 3 \\
Sangrado perigastrostomía & 2 \\
Alteración en el dispositivo de alimentación & 2 \\
Dilatación de la gastrostomía & 1 \\
Secreción serosa por la gastrostomía & 1 \\
\hline
\end{tabular}

gastrostomía ni con el procedimiento. Todas se consideraron consecuencia de la enfermedad de base que fue, en seis pacientes, cardiopatía compleja, y en otro, enfermedad neurológica; el tercer paciente fallecido padecía una malformación anorrectal y, meses después de la gastrostomía, se hizo cierre de colostomía y presentó compromiso respiratorio, infeccioso y renal con un resultado fatal.

En $13(27 \%)$ pacientes fue necesario cambiar el primer dispositivo, en 12, por disfunción del dispositivo, y en uno, por requerir una sonda gastroentérica. En siete de ellos, el cambio se hizo en los primeros dos meses después de la gastrostomía inicial (el más temprano, a los 12 días). Todos los dispositivos cambiados eran de retenedor inflable. Uno de estos procedimientos se hizo bajo anestesia general, para recanalizar y dilatar la gastrostomía.

\section{Discusión}

La gastrostomía por laparoscopia tiene múltiples ventajas en comparación con la gastrostomía abierta y con la endoscópica, algunas de las cuales se resaltan a continuación.

Las ventajas de la gastrostomía laparoscópica sobre la gastrostomía abierta son:

- Produce un mejor resultado estético porque la cicatriz queda en el ombligo ${ }^{7}$. 
- Las asas intestinales se manipulan menos y, por lo tanto, es menos prolongado el íleo posoperatorio; por consiguiente, se puede iniciar la vía entérica en forma más temprana ${ }^{8}$.

- Se producen menos adherencias peritoneales que pueden causar obstrucción intestinal.

- La incidencia de infección del sitio operatorio superficial es menor ${ }^{9}$.

- La estancia hospitalaria es menor ${ }^{7,8}$.

- $\quad$ El tiempo quirúrgico es más corto ${ }^{10}$.

Las ventajas de la gastrostomía laparoscópica sobre la gastrostomía percutánea por endoscopia son:

- Se elimina el riesgo de lesión esofágica por el endoscopio o por el retenedor de la sonda ${ }^{11}$.

- No se requiere endoscopia ni anestesia general para cambiar la sonda de la gastrostomía ${ }^{12,13}$.

- Se evita el riesgo de lesiones del colon o del intestino delgado ${ }^{12,14}$.

- Se puede escoger mejor el sitio de la pared gástrica y de la pared abdominal para hacer la gastrostomía, lo cual permite disminuir la tensión de la fijación entre ellas, haciendo que el riesgo de desalojo del botón del estómago sea menor ${ }^{12,13}$.

- Es especialmente útil en niños con un peso menor de $2 \mathrm{~kg}$, en los cuales la manipulación transesofágica puede ser más riesgosa ${ }^{12}$.

- Permite un acceso seguro durante el procedimiento, en pacientes con enfermedades de esófago y de cuello, especialmente neoplasias ${ }^{15-17}$.

Todo lo anterior demuestra que la gastrostomía por laparoscopia es un procedimiento seguro, lo cual hace que sea más llamativo que los demás procedimientos utilizados en la población pediátrica.

En este grupo de pacientes se utilizó la técnica de miniestoma para practicar la gastrostomía por laparoscopia, la cual solo requiere instrumental básico de laparoscopia y permite colocar el botón de gastrostomía de balón o el de retenedor elástico. No se documentaron complicaciones mayores intraoperatorias, ni posoperatorias. En 19 (40 \%) pacientes, se presentaron complicaciones asociadas con el cuidado de la gastrostomía, que es menor al 62,5\% reportado en la literatura científica ${ }^{3}$.

En el presente reporte, la mediana del tiempo de inicio de la nutrición entérica fue de un día. McCarter, et al., compararon dos grupos de pacientes adultos con gastrostomía endoscópica percutánea; en uno, iniciaron la nutrición a las cuatro horas y, en el otro, a las 24 , sin que se presentaran diferencias en cuanto a las complicaciones ${ }^{18}$; esto apoyaría la conducta de iniciar la nutrición entérica más tempranamente.

Un alto porcentaje de los pacientes que requieren gastrostomía tienen enfermedades de base, como cardiopatías congénitas, neuropatías o ambas, lo cual también ha sido observado por otros autores ${ }^{1,19}$.

La falla del retenedor puede causar desalojo del botón; esto sucedió en un paciente de esta serie, por lo cual requirió su recolocación bajo anestesia general en el primer mes. Esta complicación es más frecuente con los dispositivos de retenedor de balón que en los de retenedor elástico ${ }^{20}$, lo que le confiere una ventaja a los elásticos si se dispone de ellos en tamaños acordes con la edad del paciente.

En la presente serie, la mortalidad no se asoció directamente con el procedimiento o la gastrostomía; las muertes se consideraron consecuencia de la enfermedad de base. Este mismo resultado lo observaron en el estudio de Norén, et al. ${ }^{21}$.

Las técnicas de gastrostomía han tenido una interesante evolución, en la que se busca practicar procedimientos cada vez más seguros y menos invasivos, colocando dispositivos que pueden ofrecer las mayores ventajas.

Los hallazgos en esta serie de pacientes permiten afirmar que la gastrostomía laparoscópica practicada mediante la técnica de miniestoma es un procedimiento seguro, el cual se puede llevar a cabo en pacientes pediátricos con diversas enfermedades cardiacas y neurológicas. 


\title{
Experience with the miniopen laparoscopic gastrostomy technique in children
}

\begin{abstract}
Introduction: The goal of the present study is to describe the experience of laparoscopic gastrostomy using the mini-open technique with some modifications at two general hospitals in Bogotá, Colombia.

Materials and methods: A case study was conducted within the group of pediatric patients (0-13 years old) who underwent laparoscopic gastrostomy using the mini-open technique in the period August 2013 to January 2015. Pre, trans, and postoperative facts that could affect patient safety are described.

Results: 47 gastrostomies were conducted on patients aged 1 month to 13 years of age. The main indication for gastrostomy was suction-swallowing disorder (44/47, 93\%). The comorbidities presented by these patients were $85 \%$ neurological and 57\% cardiac. Most of the devices were balloon inflatable retainer devices (89.36\%). The initiation of enteral nutrition for most patients was within the first 24 hours without documented related complications. The mean time for the procedure was 57 minutes. Only minor postoperative complications were documented.
\end{abstract}

Discussion: Laparoscopic gastrostomy has multiple advantages and is a safe, reproducible procedure that requires only basic laparoscopic instruments: it is recommended as the procedure of choice for the pediatric population.

Key words: Gastrostomy; laparoscopy; pediatrics; enteral nutrition; postoperative complications.

\section{Referencias}

1. Wragg RC, Salminen H, Pachl M, Singh M, Lander A, Jester I, et al. Gastrostomy insertion in the 21st century: PEG or laparoscopic? Report from a large single-centre series. Pediatr Surg Int. 2012;28:443-8.

2. Gauderer MW, Ponsky JL, Izant Jr RJ. Gastrostomy without laparotomy: A percutaneous endoscopic technique. J Pediatr Surg. 1980;15:872-5.

3. Akay B, Capizzani TR, Lee AM, Drongowski RA, Geiger JD, Mychaliska GB. Gastrostomy tube placement in infants and children: Is there a preferred technique? J Pediatr Surg. 2010;45:1147-52.

4. Edelman DS, Unger SW, Russin DR. Laparoscopic gastrostomy. Surg Laparosc Endosc Percutan Tech. 1991;1:251-3.

5. Adams S, Mahomed A. Advances in gastrostomy placement and care in children. In: Kohout P, editor. Gastrostomy. Rijeka, Croatia: InTech; 2011. p. 3-16.

6. Baker L, Emil S, Baird R. A comparison of techniques for laparoscopic gastrostomy placement in children. J Surg Res. 2013;184:392-6.

7. Mizrahi I, Garg M, Divino CM, Nguyen S. Comparison of laparoscopic versus open approach to gastrostomy tubes. JSLS. 2014;18:28.

8. Collins JB, Georgeson KE, Vicente Y, Hardin WD. Comparison of open and laparoscopic gastrostomy and fundoplication in 120 patients. J Pediatr Surg. 1995;30:1065-71.
9. Kiran RP, El-Gazzaz GH, Vogel JD, Remzi FH. Laparoscopic approach significantly reduces surgical site infections after colorectal surgery: Data from national surgical quality improvement program. J Am Coll Surg. 2010;211:232-8.

10. Murayama KM, Schneider PD, Thompson JS. Laparoscopic gastrostomy: A safe method for obtaining enteral access. J Surg Res. 1995;58:1-5.

11. Seguel F, Ollero JC, Morato P, Rollan V, Álvarez M. Experience in performance of percutaneous endoscopic gastrostomy in 60 children. Cir Pediatr. 2003;16:125-7.

12. Zamakhshary M, Jamal M, Blair GK, Murphy JJ, Webber EM, Skarsgard ED. Laparoscopic vs. percutaneous endoscopic gastrostomy tube insertion: A new pediatric gold standard? J Pediatr Surg. 2005;40:859-62.

13. Castagnetti M, Patel S. A simple adjunct for safer change of PEG. Pediatr Surg Int. 2006;22:274-6.

14. Hermanowicz A, Matuszczak E, Komarowska M, Jarocka- Cyrta E, Wonjar J, Debek W, Matysiak K, Klek S, Laparoscopyassisted percutaneous endoscopic gastrostomy enables enteral nutrition even in patients with distorted anatomy. World J Gastroenterol. 2013;19:7696.

15. Lydiatt DD, Murayama KM, Hollins RR, Thompson JS. Laparoscopic gastrostomy versus open gastrostomy in head and neck cancer patients. Laryngoscope. 1996;106:407-10. 
16. Ho H, Ngo H. Gastrostomy for enteral access. Surg Endosc. 1999;13:991-4.

17. Bankhead RR, Fisher CA, Rolandelli RH. Gastrostomy tube placement outcomes: Comparison of surgical, endoscopic, and laparoscopic methods. Nutr Clin Pract. 2005;20:607-12.

18. McCarter TL, Condon SC, Aguilar RC, Gibson DJ, Chen YK. Randomized prospective trial of early versus delayed feeding after percutaneous endoscopic gastrostomy placement. Am J Gastroenterol. 1998;93:419-21.

19. Fascetti-Leon F, Gamba P, Dall'Oglio L, Pane A, Luigi de Angelis G, Bizzarri B, Fava G, Maestri L, Cheli M, Di Nardo $\mathrm{G}$, Complications of percutaneous endoscopic gastrostomy in children: Results of an Italian multicenter observational study. Dig Liver Dis. 2012;44:655-9.
20. Salamanca E. Tiempo útil del botón de gastrostomía de retenedor elástico $V s$. tubo de gastrostomía con retenedor inflable en niños. Experimento clínico aleatorizado (tesis). Bogotá: Pontificia Universidad Javeriana; 2013. Fecha de consulta: junio y julio de 2015. Disponible en: https://repository.javeriana.edu.co/ handle/10554/13707.

21. Norén E, Gunnarsdottir A, Hanséus K, Arnbjörnsson E. Laparoscopic gastrostomy in children with congenital heart disease. J Laparoendosc Adv Surg Tech. 2007;17:483-9.

Correspondencia: Andrea Suárez

Correo electrónico: a.suarezg@javeriana.edu.co Bogotá, D.C. 